\title{
Osteoporosis in primary biliary cirrhosis revisited
}

\author{
J Newton, R Francis, M Prince, O James, M Bassendine, D Rawlings, D Jones
}

\begin{abstract}
Background-Primary biliary cirrhosis (PBC) is increasingly being diagnosed in the earlier non-cholestatic stages of disease. Accepted wisdom has been that PBC is frequently complicated by osteoporosis. Whether this association holds true for the broader spectrum of PBC patients now recognised has not as yet been studied.

Aims-To examine the extent to which osteoporosis occurs more commonly in PBC patients than in normal individuals of the same age and sex.

Design-Retrospective review of a large cohort of well characterised PBC patients. Patients-A total of 272 PBC patients with definite or probable PBC followed up for a mean of 10.1 years (total follow up 2726 patient years) who had at least one bone mineral density measurement (BMD).
\end{abstract}

Results-In this unselected group of PBC patients, mean $\mathrm{Z}$ scores (number of SDs from age and sex matched normal mean values) at the neck of femur (NOF) and lumbar spine (LS) at first BMD measurement (7 (6) years after PBC diagnosis) were -0.1 (1.4) and $0.1(1.4)$, respectively. At first BMD measurement, 18 PBC patients had $\mathrm{Z}$ scores less than $\mathbf{- 2 . 0}$ and 85 had $T$ scores less than -2.5 . No factors predictive of osteoporosis were found in affected patients. A total of 957 BMD measurements were performed $(0.35$ per patient year of follow up); 220 patients had two or more measurements. No patient went on to develop de novo osteoporosis during follow up. In the 51 patients (who were clinically representative of the whole group) who received no PBC or bone related treatment during follow up, $\%$ BMD changes per year at the NOF and LS were $-1.6(3.2)$ and 0.1 (2.2), respectively. No variance in this "natural" rate of BMD measurement was seen in patients receiving $P B C$ modulating agents (including prednisolone and UDCA) or osteoporosis prophylaxis/therapy. Significant improvement at the LS was seen in patients undergoing liver transplantation. Conclusions-Osteoporosis is not a specific complication of PBC.

(Gut 2001;49:282-287)

Keywords: liver cirrhosis; primary biliary cirrhosis; osteoporosis

Dr D Jones, Centre for Liver Research, School of Clinical Medical Sciences, Medical School, Framlington Place,

Newcastle upon Tyne

NE2 $4 \mathrm{HH}$, UK.

D.E.J.Jones@ncl.ac.uk

Accepted for publication 31 January 2001

Primary biliary cirrhosis (PBC) is an autoimmune disease of the liver characterised by destruction of the small intrahepatic bile ducts. ${ }^{1}$ Loss of bile ducts leads to the clinical features of cholestasis. ${ }^{2}$ The disease is typically progressive, with a proportion of patients going on to develop cirrhosis. ${ }^{2}$ The rate of progression to cirrhosis can differ markedly between individual patients. ${ }^{3}$ PBC is significantly commoner in the female population and typically develops in the middle years of life. ${ }^{4}$ In most centres the median age for presentation is in the range 55-60 years (a considerable number of patients $\left(39 \%\right.$ in our series $\left.{ }^{5}\right)$ first presenting over the age of 65 years).

Osteoporosis is defined as a progressive systemic skeletal disease characterised by low bone mass and microarchitectural deterioration of bone tissue with a consequent increase in bone fragility and susceptibility to fracture. ${ }^{6}$ The World Health Organisation has defined osteoporosis quantitatively as a bone density more than 2.5 SDs below the mean value for young adults ( $\mathrm{T}$ scores less than -2.5 ). ${ }^{7}$

Osteoporosis and osteomalacia are described as complicating PBC, ${ }^{89}$ and the scenario of a cholestatic disease with its peak occurrence in middle aged and older women is certainly one which would raise concerns regarding the risks of osteoporosis development. Suggested causes for osteoporosis in PBC include impaired calcium and vitamin $\mathrm{D}$ absorption resulting from profound cholestasis, hyperbilirubinaemia impairing osteoblast function, and corticosteroid use. $^{1011}$

The prevalence of osteoporosis increases however with advancing age in all subject groups, ${ }^{12}$ raising the possibility that the presence of cases of osteoporosis in PBC populations might result not from a disease associated pathological process but simply from the age and sex of the affected individuals. Data to quantify the risk of osteoporosis and associated fracture in PBC are limited. Bone density measurements in three small studies have suggested a specific association between osteoporosis and PBC. ${ }^{10}{ }^{13}{ }^{14}$ Conversely, a further study in 47 patients ${ }^{15}$ suggested that PBC is not complicated by osteoporosis. Data regarding bone histology in PBC are equally contradictory. ${ }^{131416}$ Although the use of oral corticosteroids may potentially increase the risk of osteoporosis in PBC, data to demonstrate an additive risk are limited. ${ }^{315}$

With improvements in diagnosis, the clinical spectrum of PBC has changed over the past 20 years, from a disease predominantly presenting with severe cholestasis to one which is typically diagnosed in its early asymptomatic stages. ${ }^{3}{ }^{17}$ This change in clinical spectrum away from overt cholestasis may further challenge the
Abbreviations used in this paper: $\mathrm{PBC}$, primary biliary cirrhosis; BMD, bone mineral density; LS, lumbar spine; NOF, neck of femur; DEXA, dual energy $x$ ray absorptiometry; HRT, hormone replacement therapy. 
assumption that the majority of patients with PBC are at risk of osteoporosis. With the development of effective treatment for osteoporosis it is clearly important however to identify patients who are at high risk of developing the condition. We therefore set out to address the question: are patients with $\mathrm{PBC}$ at extra risk of osteoporosis? In order to do this we have carried out a pragmatic retrospective examination of bone density measurements in a large group of (otherwise) unselected PBC patients.

\section{Methods}

BONE DENSITY MEASUREMENTS

All patients attending the Regional Medical Physics Department, Newcastle, UK, for bone mineral density measurement (BMD) have diagnostic and clinical details recorded. All patients having BMD with a recorded diagnosis of PBC since the start of the Newcastle BMD service were identified retrospectively. To date, no systematic protocols have been in place in Newcastle for BMD measurement in subjects with PBC. Decisions regarding the timing and frequency of BMD measurements analysed in this study were made by individual clinicians on the basis of perceived need.

Bone density measurements $\left(\mathrm{g} / \mathrm{cm}^{2}\right)$ of the femoral neck (NOF) and lumbar spine (LS) were taken in PBC patients using dual energy $x$ ray absorptiometry (DEXA). The BMD service upgraded its equipment from a Hologic QDR-1000 to a Hologic QDR-2000 DEXA machine in 1992; 94\% of BMD measurements were taken using the newer equipment but validation experiments have shown comparable results with the two machines. ${ }^{18}$ Patients were scanned according to the manufacturer's recommendations. Scans were analysed, at the time of performance, by trained and experienced operators in accordance with standard procedures. The in vivo precision measurement at the spine and femoral neck is $1-2 \%$. Individual $\mathrm{BMD}$ results were obtained as a real density in $\mathrm{g} / \mathrm{cm}^{2}$ but have been compared with the normal sex specific ranges for young adults and the age related range to give $T$ and $Z$ scores, respectively.

$\mathrm{T}$ and $\mathrm{Z}$ scores were calculated according to established international normal ranges. The $\mathrm{T}$ score is the number of SD units above or below the mean for normal young adults while the $Z$ score is the number of SD units above or below the age related normal mean. ${ }^{7}$ Percentage change per year was calculated as a linear regression of $\mathrm{BMD}$ on time and indicated the change in BMD measurement that occurred per year.

PBC PATIENTS

The hospital case records of all patients in whom a diagnosis of PBC was considered in the Northern Region were examined and clinical, biochemical, and liver histology data recorded on a regional PBC database.${ }^{19}$ Details were reviewed for all those individuals who had attended for BMD measurement with a clinical diagnosis of PBC $(n=317)$. Treatment details were reviewed from the case notes for all those who had repeated BMD measurements. Ethics permission for the collection of epidemiological data and development of the database was obtained from the Joint Newcastle Hospitals/ University Ethics Committee and all other ethics committees in the whole study region.

All patients with definite and probable PBC who had undergone BMD measurement were identified. A definite diagnosis of PBC was made on the basis of the presence of all three of: (a) antimitochondrial antibody titre $\geqslant 1: 40$, (b) cholestatic liver function tests, and (c) compatible or diagnostic liver histology. A diagnosis of probable PBC was made when two of these parameters were present. ${ }^{20}$ The date at which a presumed diagnosis of PBC was made was the time at which two of the above three diagnostic criteria were first fulfilled and recorded in the case records. The date of presumed diagnosis was thus regarded as the date of "presentation" of disease.

As biochemical results may be from different laboratories with varying reference ranges, the data were expressed as a ratio of the normal range. A value within the normal range is defined as being 1 while any result outside the normal range is expressed as a ratio. For bilirubin, transaminases, and alkaline phosphatase, this ratio is the result/upper limit of normal (for example, abnormal bilirubin value $>1$ ) while with albumin it is value/lower limit of normal (for example, abnormal result $<1$ ). All liver biopsies were reviewed for this study by one of two histopathologists and classified according to current histological criteria into "early" (Scheuer stage I/II ) and "advanced" (Scheuer stage III/IV). ${ }^{2}$

\section{STATISTICAL ANALYSIS}

Results are expressed as mean (SD) or median (range). Comparisons between means were carried out using the Student's unpaired and paired $t$ test. The $\chi^{2}$ test and Mann-Whitney $\mathrm{U}$ test were used where appropriate. Correlations were carried out using the Spearman rank correlation coefficient. A result was considered statistically significant when $\mathrm{p}<0.05$.

\section{Results}

A total of 317 patients were identified who had at least one BMD measurement performed, and in whom a diagnosis of PBC had been recorded in the clinical extract. Cross reference with the PBC database confirmed that 272 of these individuals had definite or probable PBC (definite $199(73 \%)$ ). The remaining 45 patients did not fulfil the diagnostic criteria for PBC or were found to have other liver diseases after review of the medical case records. These patients were excluded from further analysis. During the period when BMD measurement was available, 415 patients were being followed up in the PBC clinic and therefore were available to be referred for BMD measurement.

In the first part of this study we examined the initial BMD measurements for the whole PBC population. In the second we identified those PBC patients who had gone on to have at least one further follow up BMD measurement, and examined these retrospectively with regard to treatment and changes in $\mathrm{T}$ and $\mathrm{Z}$ scores. 
FIRST SCANS IN PATIENTS WITH PBC WHO HAD BMD MEASUREMENT

The 272 individuals with definite or probable PBC who had at least one BMD measurement performed had, by the audit point for the current study, been followed up for a total of 2726 years (mean (SD) follow up 10.1 (5.3) years; range $1-29$ ).

A total of $255(94 \%)$ patients were female. Of this female group, 45 (18\%) had undergone a hysterectomy and 163 (64\%) were postmenopausal (mean (SD) age at menopause 48.1 (4.5) years).

Mean (SD) age at first diagnosis was 56 (11) years, with $115(42 \%)$ subjects presenting for the first time over the age of 65 . Mean age at the time of first BMD measurement was 62 (11) years and the mean (SD) time from presentation to first BMD measurement was 7 (6) years. Fifty patients died from all causes during follow up, $19 / 50(38 \%)$ from liver related causes.

The results of the initial BMD measurements for the whole PBC patient group are shown in table 1 . The most striking observation is that mean (SD) $\mathrm{Z}$ scores for the NOF $(-0.1$ (1.4)) and LS (0.1 (1.4)) did not differ significantly from 0 (that is, there was no significant difference between the mean values for BMD measurements in PBC patients and those expected for an age and sex matched "normal" population).

Biochemical markers of PBC severity (bilirubin and albumin) and other liver related biochemical tests (alkaline phosphatase and transaminase) at the time of initial BMD measurement were examined (table 1). No correlation was seen between first NOF or LS BMD absolute value and bilirubin level. No correlation was seen between bilirubin level and initial $\mathrm{T}$ or $\mathrm{Z}$ score at either the NOF or LS.

Liver biopsy samples were available for systematic review in 155 patients. Eighty three of $155(54 \%)$ patients had late disease (stage III/IV) and 72/155 (46\%) early disease on their index liver biopsy. There was no significant difference in $\mathrm{Z}$ or $\mathrm{T}$ scores at either the NOF or

Table 1 Liver function tests (ratio of upper limit of normal) and initial bone mineral density measurements in the total unselected group of primary biliary cirrhosis patients $(n=272)$ and in those with $T$ score less than and more than -2.5

\begin{tabular}{|c|c|c|c|}
\hline & $\begin{array}{l}\text { Total group } \\
(n=272)\end{array}$ & $\begin{array}{l}T \text { score below }-2.5 \\
(n=85)\end{array}$ & $\begin{array}{l}T \text { score above }-2.5 \\
(n=187)\end{array}$ \\
\hline Age at 1st scan (y) & (11) & $(8)^{\star}$ & $(11)^{\star}$ \\
\hline \multicolumn{4}{|c|}{ Liver function tests (ratio of normal) } \\
\hline Bilirubin & $1.34(1.09)$ & $1.34(1.1)$ & $1.35(1.13)$ \\
\hline Albumin & $0.99(0.05)$ & $0.98(0.06)$ & $0.99(0.06)$ \\
\hline Alk phos & $3.39(3.13)$ & $3.8(3.8)$ & $3.34(3.29)$ \\
\hline Transaminase & $2.02(2.03)$ & 1.9 (1.2) & $2.0(2.1)$ \\
\hline \multicolumn{4}{|l|}{ NOF } \\
\hline $\operatorname{BMD}\left(\mathrm{g} / \mathrm{cm}^{2}\right)$ & $0.7 \quad(0.2)$ & $0.6(0.1)$ & $0.75(0.19)$ \\
\hline T score & $-1.8 \quad(1.6)$ & - & - \\
\hline Z score & $-0.1 \quad(1.4)$ & - & - \\
\hline \multicolumn{4}{|l|}{ LS } \\
\hline $\mathrm{BMD}\left(\mathrm{g} / \mathrm{cm}^{2}\right)$ & $1.1 \quad(0)$ & $0.8 \quad(0.2)$ & $0.96(0.14)$ \\
\hline $\mathrm{T}$ score & -1.3 & - & - \\
\hline$Z$ score & $0.1 \quad(1.4)$ & - & - \\
\hline
\end{tabular}

All values are mean $(\mathrm{SD})$.

NOF, neck of femur; BMD, bone mineral density; LS, lumbar spine; Alk phos, alkaline phosphatase.

${ }^{\star} \mathrm{p}<0.05$.
LS between those patients with early disease and those with advanced disease.

Eighty five of 272 PBC patients had osteoporosis (according to the WHO definition of a $\mathrm{T}$ score of less than -2.5 ) at the time of initial BMD measurement. Mean serum bilirubin and albumin levels were the same in these patients as in the whole PBC patient group (table 1), suggesting that this is not a group of patients suffering from more cholestatic disease. As might be expected, the group of patients found to have osteoporosis at the time of their first BMD measurement had a higher mean (SD) age than the nonosteoporotic patients (67 (8) v 59 (11) years; $\mathrm{p}<0.05)$. Fifty two of 85 patients with osteoporosis on their initial BMD had undergone a liver biopsy. Thirty of $52(57 \%)$ patients had histologically advanced disease and 22 (43\%) early disease. Strikingly, among the 17 male PBC patients in the study, nine (52\%) presented with an initial $\mathrm{T}$ score of less than -2.5 and were thus osteoporotic.

Among the 272 PBC patients, 18 (6.6\%; 15 female, three male) had significantly lower BMD than would be predicted for their age and sex (defined as $\mathrm{Z}$ score less than -2.0 ; see clinical details in table 2). Only $3 / 18$ had elevated serum bilirubin levels at the time of their index scan, again suggesting that more severe cholestasis was unlikely to be the explanation for the lower than predicted BMD values seen in this group.

CHANGES IN BMD MEASUREMENT OVER TIME IN PATIENTS HAVING MULTIPLE ASSESSMENTS

A total of 957 scans were performed on 272 patients with definite or probable PBC (equivalent to $0.35(957 / 2726)$ scans per patient year of follow up). Mean (SD) number of scans per patient was 5 (0.5). Median number of scans per subject was 5 (range 1-11). Elevation of bilirubin ratio, length of follow up, and age at first scan were found to be associated with patients having multiple scans $(\mathrm{p}<0.005$, $\mathrm{p}<0.01$, and $\mathrm{p}<0.01$, respectively). A total of 225/272 (82.7\%) PBC patients had two or more scans performed (repeat scanning being performed at the discretion of the managing clinician).

Medical notes were reviewed for details of treatments received by each individual in whom repeated BMD measurements had been performed. Full treatment details were available for 220 of 225 patients having multiple scans.

Among the 225 patients who underwent multiple BMD measurements, none who had a $T$ score greater than -2.5 on their index scan $(n=141)$ went on to develop osteoporosis (using the WHO definition) during follow up. Only one PBC patient who did not have a $\mathrm{Z}$ score greater than -2.0 on their index scan went on to develop a $\mathrm{Z}$ score lower than -2 at NOF during follow up (and this individual in fact had a $\mathrm{Z}$ score of -2 at the LS on their index scan). 
Table 2 Clinical details of patients with $Z$ scores less than -2 on the index scan at either the neck of femur or lumbar spine ( $n=18$ )

\begin{tabular}{|c|c|c|c|c|c|c|c|c|c|c|c|c|}
\hline \multirow[b]{2}{*}{ Patient No } & \multirow[b]{2}{*}{$\begin{array}{l}\text { Age at } 1^{\text {st }} \\
\text { scan }\end{array}$} & \multirow[b]{2}{*}{$\operatorname{Sex}$} & \multirow[b]{2}{*}{ Dead } & \multirow[b]{2}{*}{ Transplant } & \multirow[b]{2}{*}{ Steroid } & \multirow[b]{2}{*}{$U D C A$} & \multirow[b]{2}{*}{ Bisphos } & \multirow[b]{2}{*}{ Ca suppl } & \multirow[b]{2}{*}{ Biopsy } & \multicolumn{3}{|c|}{ Liver function tests (ratio of normal) } \\
\hline & & & & & & & & & & Bilirubin & Alk phos & Transminase \\
\hline 1 & 60 & $\mathrm{~F}$ & & & & $\mathrm{Y}$ & $\mathrm{Y}$ & $\mathrm{Y}$ & B & $<1$ & 2.1 & $<1$ \\
\hline 2 & 61 & $\mathrm{~F}$ & & & $\mathrm{Y}$ & & $\mathrm{Y}$ & $\mathrm{Y}$ & A & $<1$ & 1.7 & 4.6 \\
\hline 3 & 58 & $\mathrm{~F}$ & & & $\mathrm{Y}$ & Y & Y & & & $<1$ & 4 & $<1$ \\
\hline 4 & 59 & $\mathrm{~F}$ & & & & & $\mathrm{Y}$ & & & $<1$ & $<1$ & $<1$ \\
\hline 5 & 73 & $\mathrm{~F}$ & & & & $\mathrm{Y}$ & $\mathrm{Y}$ & $\mathrm{Y}$ & B & 1.3 & 3.2 & 1.4 \\
\hline 6 & 48 & $\mathrm{~F}$ & & $\mathrm{Y}$ & & & & & & $<1$ & 1.3 & 1.9 \\
\hline 7 & 64 & $M$ & & & & $\mathrm{Y}$ & $\mathrm{Y}$ & & & $<1$ & 5.2 & 5.1 \\
\hline 8 & 67 & $\mathrm{~F}$ & $\mathrm{Y}$ & & & $\mathrm{Y}$ & $\mathrm{Y}$ & $\mathrm{Y}$ & A & $<1$ & $<1$ & $<1$ \\
\hline 9 & 53 & $\mathrm{~F}$ & & & & & $\mathrm{Y}$ & & A & $<1$ & 1.9 & 2 \\
\hline 10 & 70 & $\mathrm{~F}$ & & & & $\mathrm{Y}$ & & & A & $<1$ & 5.9 & 1.6 \\
\hline 11 & 62 & M & & & & & & & A & $<1$ & 3.3 & $<1$ \\
\hline 12 & 69 & $\mathrm{~F}$ & $\mathrm{Y}$ & & & & & & & $<1$ & 11.5 & 3.8 \\
\hline 13 & 54 & $\mathrm{~F}$ & & Y & & & & & & $<1$ & $<1$ & $<1$ \\
\hline 14 & 64 & $\mathrm{~F}$ & & $\mathrm{Y}$ & & & & & & $<1$ & 7.5 & 5.8 \\
\hline 15 & 58 & $M$ & & $\mathrm{Y}$ & & & & & A & $<1$ & 7.6 & 4.5 \\
\hline 16 & 58 & $\mathrm{~F}$ & $\mathrm{Y}$ & & & & & $\mathrm{Y}$ & A & 1.7 & 6.7 & 2.2 \\
\hline 17 & 65 & $\mathrm{~F}$ & $\mathrm{Y}$ & & & & & $\mathrm{Y}$ & & $<1$ & 1.2 & $<1$ \\
\hline 18 & 67 & $\mathrm{~F}$ & & & & $\mathrm{Y}$ & $\mathrm{Y}$ & & & 2.6 & 2.9 & 4.4 \\
\hline
\end{tabular}

Bispho, bisphosphonate; Alk phos, alkaline phosphatase; Y, yes; N, no; dead, died during follow up; Ca suppl, calcium supplements; B, early histological disease; A, advanced histological disease.

Table 3 Liver function tests (ratio of upper limit of normal) and bone mineral density measurements for the untreated primary biliary cirrhosis patient group (those patients who had undergone repeated scans but received no liver or bone related treatment $)(n=51)$

\begin{tabular}{lc}
\hline & Untreated group $(n=51)$ \\
\hline Liver function tests (ratio of normal) \\
Bilirubin & $1.2(1.0)$ \\
Albumin & $1.0(0.01)$ \\
Alk phos & $2.1(1.5)$ \\
Transminase & $1.7(2.1)$ \\
NOF & \\
BMD $\left(\mathrm{g} / \mathrm{cm}^{2}\right)$ & $0.7(0.2)$ \\
T score & $-1.8(1.6)$ \\
Z score & $-0.1(1.4)$ \\
$\%$ change/year & $-1.6(3.2)$ \\
LS & $1.0(0.2)$ \\
BMD $\left(\mathrm{g} / \mathrm{cm}^{2}\right)$ & $-0.8(1.6)$ \\
T score & $0.6(1.6)$ \\
Z score & $0.1(2.2)$ \\
\% change/year &
\end{tabular}

All values are mean $(\mathrm{SD})$.

Alk phos, serum alkaline phosphatase; NOF, neck of femur; $\mathrm{BMD}$, bone mineral density; LS, lumber spine.

The untreated group: those having multiple assessments who had received no bone or liver modulating therapy

Fifty one of 220 patients (23\%) in the follow up scan group for whom full details were available had not received any treatment pertinent to their liver disease, or potentially bone altering medication, during the follow up period. This group will subsequently be referred to as the untreated group. The remaining 169 patients are referred to as the treated group (see below). BMD measurements and liver function tests for the untreated group are shown in table 3. Comparable proportions of early and advanced disease patients were seen in the untreated and treated groups $(38 \%$ v $43 \%$ early disease, respectively).

Effect of bone modulating treatment on those having multiple assessments

Treatments studied were: (a) corticosteroids, (b) biphosphonates, (c) calcium and vitamin D supplements, and (d) hormone replacement therapy (HRT).

The remaining 169 patients were treated with one or more of the above treatment modalities during follow up. Thirty had received steroids $(30 / 220(13.6 \%)), 51$ biphosphonates (51/220 (23\%)), 13 HRT $(13 / 220$ $(5.9 \%))$, and 66 calcium supplements $(66 / 220$ $(30 \%))$. In addition, 21 patients had undergone orthoptic liver transplantation (21/ $220(9.5 \%))$.

In those who had repeated BMD measurement while continuously being treated with one or more of the above treatments - that is, treatment had been started prior to the first BMD measurement-percentage change per year in BMD was compared with percentage changes measured in the untreated group. No significant differences in percentage change per year (measured at both the NOF and LS) were seen between patients treated with biphosphonates, HRT, prednisolone, or calcium supplements and the untreated control group.

Of the patients who underwent orthotopic liver transplantation during the follow up period, 16/21 had BMD measurements taken before and after transplantation. Although the $\mathrm{Z}$ scores taken at the NOF were not significantly different, those at the LS increased significantly after transplantation (mean (SD) -1.162 (1.057) before transplantation compared with $-0.475(0.859)$ after transplantation; $\mathrm{p}<0.0001$ ).

\section{Discussion}

In this study, we have presented the largest series of bone density measurements reported in patients with PBC to date. Although the patients constituted a heterogenous group with respect to the treatment they had received, our data showed that osteoporosis was no more prevalent in this large population of PBC patients than would have been expected in a normal population of comparable age and sex. This strongly suggests that while osteoporosis occurs in a proportion of patients, it is not a specific complication of PBC.

Previous studies addressing the question of osteoporosis in PBC have drawn different conclusions. This may be due to the numbers of patients included (typically fewer than 50 in 
the earlier studies) or the case mix of patients with PBC (typically more skewed towards advanced disease at a time when liver transplantation was not routinely available in earlier studies). It is essential that conclusions regarding the prevalence of osteoporosis in PBC be drawn from the largest possible patient population. It is also important when considering a disease that is common in older people that age matched values be used to draw any conclusions regarding the added risk of osteoporosis resulting from the disease state. It has recently been shown that rather than being a disease of young and middle aged women, PBC is actually a disease that often presents for the first time over the age of $65 .^{5}$ It is not surprising that osteoporosis is not uncommon in a disease predominately affecting peri- or postmenopausal women. It is important to appreciate however that the apparent risk of osteoporosis in PBC reflects the demographics of the disease population rather than a specific disease process. It has previously been noted that the duration of PBC was significantly longer in those patients with osteoporosis compared with those without. ${ }^{13}$ This may similarly be interpreted that as older people have had $\mathrm{PBC}$ for longer, osteoporosis in PBC is an age related phenomenon rather than one resulting from $\mathrm{PBC}$ specific disease processes.

The group who had osteoporosis ( $\mathrm{T}$ score below -2.5) at the time of the first BMD measurement was, on average, significantly older than the PBC group as a whole, again underlining the fact that osteoporosis is more a disease of older people than a specific complication of PBC. A large proportion (52\%) of men with PBC had osteoporosis at presentation. The reasons for this are unclear and require further evaluation.

Although osteoporosis was no more prevalent in the PBC population as a whole than the normal population, and while elevated serum bilirubin was not associated with reductions in bone density, cases of osteoporosis obviously did occur. The data regarding bone density before and after liver transplantation suggest that osteoporosis is exacerbated by end stage disease (the typical disease state for patients requiring liver transplantation). This observation is fully compatible with the overall lack of association between PBC and osteoporosis when one bears in mind the relative rarity in our broad PBC population of severe, let alone end stage, disease. In this study we showed significant increases in BMD after liver transplantation when measured at the LS but not at the NOF. Changes are often slower at the NOF than at the LS and this may well account for the apparent discrepancy. This finding confirms the previous results of Eastell and colleagues ${ }^{21}$ that after an initial fall in BMD, orthotopic liver transplantation significantly increases bone density. The mechanism by which this occurs requires further evaluation but may be an indicator of general debility followed by improved mobility, or due to relief of cholestasis.

Our findings suggest (to the extent that is possible in a historical descriptive study) that drug treatment did not materially alter the development of osteoporosis. This is in contrast with the work of Stellon and colleagues ${ }^{22}$ who demonstrated significantly greater bone loss at all sites measured in those patients that were receiving, or had received, corticosteroids. Almdal et al however showed that bone mineral content was not significantly associated with duration of liver disease, impairment of liver function, variables reflecting calcium homeostasis, or previous treatment with glucocorticosteroids. ${ }^{15}$ This would appear to concur with our present findings.

In this study, we acquired evidence from a large cohort of patients with PBC. Given these data it is difficult to justify routine BMD measurement in patients with PBC, particularly as features that put individuals at risk are not readily identifiable. "Targeted" screening of only those patients with overtly cholestatic disease who, based on earlier studies might have been considered to be the at risk, would in fact have resulted in the majority of osteoporotic patients being missed. In addition, our findings regarding repeat screening suggest that following exclusion of prevalent osteoporosis by initial scan, the pick up rate of subsequent incident cases was extremely low. Therefore, there is a convincing argument that follow up scanning is inappropriate. The information from this study will be useful in developing guidelines for the appropriate assessment of osteoporosis in patients with PBC.

This study was a retrospective review of the management of patients with PBC. The weaknesses of retrospective studies of this nature are well known. Limitations notwithstanding, we believe that our observation, that osteoporosis is not a specific complication of primary biliary cirrhosis, has important implications for the future management of PBC patients.

1 Neuberger JM. Primary biliary cirrhosis. Lancet 1997;350:875-9.

2 Scheuer PJ. Primary biliary cirrhosis. Proc R Soc Med 1967; 60:1257-61.

3 Jones DEJ, James OFW, Bassendine MF. Primary biliary cirrhosis: Clinical and associated autoimmune features and natural history. Clin Liver Dis 1998;2:265-82.

4 Sherlock S, Scheuer PJ. The presentation and diagnosis of

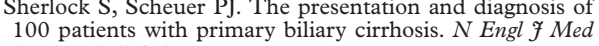
1973;289:674-8.

5 Newton JL, Jones DEJ, Metcalf JV, et al. The presentation and mortality of primary biliary cirrhosis in older patients. Age Ageing 2000;29:305-9.

6 Consensus Development Conference. Diagnosis, prophylaxis and treatment of osteoporosis. Am $\mathcal{F} \mathrm{Med} 1991 ; 90$ : 107-10.

7 WHO Study Group. Assessment of fracture risk and its application to screening for postmenopausal osteoporosis. report of a WHO study group. Geneva: World Health Organsisation, 1994.

8 Atkinson $M$, Nordin BEC, Sherlock S. Bone disease in obstructive jaundice. Q $\mathcal{F M e d}$ 1956;25:299-312.

9 Kehayoglou AK, Holdsworth CD, Agnew JE, et al. Bone Kehayoglou AK, Holdsworth CD, Agnew JE, et al. Bone
disease and calcium absorption in primary biliary cirrosis with special reference to vitamin-D therapy. Lancet 1968;i: with special 715 .

10 Hodgson SF, Rolland-Dickson E, Wahner HW, et al. Bone loss and reduced osteoblast function in primary biliary cirrhosis. Ann Intern Med 1985;103:855-60.

11 Janes CH, Rolland-Dickson E, Okazaki R, et al. Role of hyperbilirubinaemia in the impairment of osteoblast proliferation associated with cholestatic jaundice. $\mathcal{F}$ Clin Invest 1995;95:2581-6.

12 Sutcliffe AM, Francis RM. Osteoporosis: treatment options in elderly patients. Prescriber 1998;9:91-4.

13 Guanabens N, Pares A, Marasino L, et al. Factors influencing the development of metabolic bone disease in primary ing the development of metabolic bone disease in pr.

14 Matloff DS, Kaplan MM, Neer RM, et al. Osteoporosis in primary biliary cirrhosis: effects of 25 -hydroxyvitamin D3 treatment. Gastroenterology 1982;83:97-102. 
15 Almdal T, Schaadt O, Veaterdal-Jorgensen J, et al. Vitamin $\mathrm{D}$, parathyroid hormone and bone mineral content of lumbar spine and femur in primary biliary cirrhosis. F Intern Med 1989;225:207-13.

16 Mitchison HC, Malcom AJ, Bassendine MF, et al. Metabolic bone disease in primary biliary cirrhosis at presentation. Gastroenterology 1988;94:463-70.

17 James OFW, Bhopal R, Howel D, et al. Primary biliary cirrhosis once rare, now common in the UK? Hepatology 1999;30:390-4

18 Anderson FH, Francis RM, Faulkner K. Androgen supplementation in eugonadal men with osteoporosis-effects of 6 months of treatment on bone mineral density and cardiovascular risk factors. Bone 1996;90:171-7.
19 Metcalf JV, Bhopal RS, Gray J, et al. Incidence and prevalence of primary biliary cirrhosis in the city of
Newcastle-upon-Tyne, England. Int $\mathcal{F}$ Epidemiol 1997;26: 830-6.

20 Metcalf JV, Howel D, James OFW, et al. Primary biliary cirrhosis: epidemiology helping the clinician. BMF 1996; 312:1181-2

21 Eastell R, Rolland-Dickson E, Hodgson SF, et al. Rates of vertebral bone loss before and after liver transplantation in women with primary biliary cirrhosis. Hepatology 1991;14: 296-300.

22 Stellon AJ, Davies A, Compston J, et al. Osteoporosis in chronic cholestatic liver disease. Q f Med 1985;223:783-

\title{
Narrative Based Medicine, An Interdisciplinary Conference
}

\author{
Research, Narrative, and Practice
}

A two day conference-Monday 3rd and Tuesday 4th September 2001

\section{Homerton College, Cambridge, UK}

BMF Publishing Group

For full details contact: BMA/BMJ Conference Unit, Tavistock Square, London, WC1H 9JP Tel: +44 (0)20 7383 6819; fax: +44 (0)207383 6663; email: clyders@bma.org.uk.

www.quality.bmjpg.com 